Jpn J Electroph $1997 ; 41: 179$

〔Full Paper〕

\title{
Capillary affinophoresis of fluorescence-labeled pea lectin with laser-induced fluorescence detection
}

\author{
Kiyohito Shimura and Ken-ichi Kasai
}

\begin{abstract}
SUMMARY
Affinophoresis is a type of affinity electrophoresis in which an affinophore, a conjugate of an affinity ligand and a multiply charged soluble matrix, causes a mobility change in proteins that have specific affinity for the ligand. This technique enables, not only the separation of a specific molecule, but also the analysis of specific interaction between biomolecules. The affinity constant between fluorescence-labeled pea lectin and affinophores bearing mannoside as an affinity ligand, was determined by capillary electrophoresis using laser-induced-fluorescence detection. Although the labeling of the protein caused microheterogeneity as evidenced by electrophoretic mobility, the affinity constant determined for the main peak in the electropherogram of the labeled lectin agreed with those obtained for unlabeled lectin by the same type of analysis using ultraviolet-absorption detection. As a result of the use of the fluorescence detection, the consumption of the protein sample is greatly reduced to several pg per run.
\end{abstract}

Key words : affinity electrophoresis, capillary electrophoresis, laser-induced fluorescence, fluorescence labeling, affinity constants.

\section{INTRODUCTION}

The use of capillary electrophoresis (CE) for the analysis of molecular recognition has been shown for many interacting systems. ${ }^{1-3)}$ We reported the development of a type of affinity electrophoresis, affinophoresis, in which the electrophoretic mobility of a binding protein was specifically modified by interaction with an affinophore, which was produced by coupling an affinity ligand(s) to a charged soluble matrix, such as succinylpolylysine. ${ }^{4)}$ The utility of affinophoresis, in a capillary system, was demonstrated for the quantitative analysis of specific interactions between a lectin and sugars. ${ }^{5)}$

In capillary electrophoresis, the scale of separation space is approximately one thousandth that for electrophoresis carried out using gel slabs or rods. This permits a proportional reduction in sample size for $\mathrm{CE}$ analysis, as compared with the conventional electrophoresis formats using gel or membrane as supporting media. Although the sample size can be quite small, the short pass length available for on-line ultraviolet (UV) detection requires a relatively high concentration, i.e., 0.1 to $1 \mathrm{mg} / \mathrm{ml}$ for protein samples.

Laser-induced fluorescence (LIF) detection is the

志村清仁，笠井献一；Department of Biological Chemistry, Faculty of Pharmaceutical Sciences, Teikyo University. Correspondence address: Kiyohito Shimura, Department of Biological Chemistry, Faculty of Pharmaceutical Sciences, Teikyo University, Sagamiko, Kanagawa 199-01, Japan.

Abbreviations: CE, capillary electrophoresis; UV, ultraviolet ; LIF, laser-induced fluorescence ; TR, 5-carboxytetramethylrhodamine; Tris, tris (hydroxymethyl) aminomethane; IEF, isoelectric focusing.

(Received January 22, 1997, Accepted March 3, 1997, Published August 15, 1997) 
most desirable option in $\mathrm{CE}$, when samples at low concentration are to be analyzed. For this purpose, an Ar-ion laser at 488 or $512 \mathrm{~nm}$ and a He-Ne laser at $543.5,594$ or $632 \mathrm{~nm}$ are most often used. Detection with LIF is more sensitive than UV-absorption by a factor of 2-to-3 orders of magnitude, even when a simple on-capillary detector ${ }^{6)}$ and the detection limit of an optimized system, using a rectangular sheath-flow cell has been reported to be less than ten molecules. ${ }^{7)}$

To exploit the high sensitivity of LIF detection, labeling of samples with a fluorescent dye that is compatible with the wave length of the laser is necessary, but most of the labeling reactions for the case of proteins change the net charge on the protein molecule. The protein amino groups to be derivatized are charged at neutral pHs, i.e., partially charged for the case of $\mathrm{N}$-terminal amino groups and fully for the case of lysyl $\varepsilon$-amino groups. For example, when a succinimidyl ester of a carboxyl derivative of a dye is used for labeling, the ionization of the amino groups is suppressed and the number of positive charges will be reduced. Thus, the labeling reactions result in the formation of a series of proteins with different net charges, depending on the number of introduced labeling groups. $\left.{ }^{8}\right)$ The use of dyes, having net negative or positive charge(s), will also result in an additional modification in the net charge of the protein. Therefore, the heterogeneity produced as a result of labeling can pose a significant problem when such labeled proteins are used for the analysis of molecular interactions using LIF as a method of the detection.

Another issue in the use of labeled proteins is the influence of the labels on the biochemical activity of proteins. Fluorescent dyes, such as fluorescein and rhodamines, are relatively bulky and these groups might interfere with the interaction of a protein with specific molecules or cause an alterations in the protein conformation which could ultimately lead to a change in binding affinity. To evaluate the utility of labeled proteins and LIF detection for ultramicroscale analysis of molecular interaction using capillary electrophoresis, pea lectin was labeled with a fluorescent dye and were quantitatively analyzed with respect to its interactions with mannoside-bearing affinophores. The latter have been shown to be effective in the isolation of the lectin from the pea-seed extract using agarose-gel electrophoresis. $^{9)}$

\section{MATERIALS AND METHODS}

The following materials were obtained from commercial sources: $p$-aminophenyl $\alpha$-D-mannoside, poly-L-lysine (degree of polymerization of 270) (Sigma, St. Louis, Missouri) ; 5-carboxytetramethylrhodamine (TR) succinimidyl ester (Molecular Probes, Eugene, Oregon) ; BioGel P6 (BioRad Laboratories, Richmond, California) ; carrier ampholytes (Ampholine pH 3.5-10), a protein-pI marker kit (Broad pI Calibration Kit, pH 3-10), Sephadex G100, and an anion exchange column (Mono Q HR 5/5) (Pharmacia Biotech, Tokyo); fused silica capillaries (GL Sciences Inc., Tokyo). Pea lectin was isolated from Pisum sativum seeds obtained at a local seed shop using the reported procedure. $^{10)}$

\section{Preparation of mannoside affinophores}

The affinophores bearing mannoside as an affinity ligand were prepared by coupling $p$-aminophenyl $\alpha$ $D$-mannoside to a portion of the carboxyl groups of completely succinylated poly-L-lysine as described previously.9) Four $0.5 \mathrm{ml}$ aliquots of the solution of succinylpolylysine $(20 \mu \mathrm{mol}$ of lysine residue in $0.1 \mathrm{M} \mathrm{NaCl}$, adjusted to $\mathrm{pH} 5$ with $6 \mathrm{M} \mathrm{HCl}$ ) were mixed with $0,10,20$, or $40 \mu 1$ of $0.1 \mathrm{M} p$-aminophenyl $\alpha$-D-mannoside solution to give a series of affinophores which differed with respect to the density of the ligand. A solution of water-soluble carbodiimide (1-ethyl-3-(3-dimethylaminopropyl) carbodiimide hydrochloride, $1 \mathrm{M}, 20 \mu \mathrm{l}$ ) was added to each mixture and allowed to react for $30 \mathrm{~min}$ at room temperature. The reaction mixtures were separately dialyzed twice at room temperature

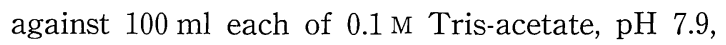
containing $0.02 \%$ sodium azide as a preservative and used as the affinophore. The concentration of the affinophore solution was determined by amino acid analysis of lysine after acid hydrolysis and that of the mannoside was determined by the phenolsulfuric acid reaction method. The affinophores were numbered from 0 to 3 in order of increasing density of the ligand. The ligand density ( $\{[\mathrm{Man}] /$ [Lys] $\times 100 \%)$ was found to be: affinophore- 0 , $0.05 \%$; affinophore-1, $4.2 \%$; affinophore-2, $9.3 \%$; 
and affinophore-3, $17.5 \%$. Most of the ligand (84 to $99 \%$ of added) were recovered in the polymer fractions.

\section{Labeling of pea lectin}

Pea lectin was purified from the seed extract using affinity chromatography on Sephadex G100 according to the reported procedure. ${ }^{10)}$ The most basic $\mathrm{pI}$ variant $(\mathrm{pI}=7.05)$ of the lectin was isolated by ion-exchange chromatography on a Mono Q HR column as previously reported. ${ }^{11)}$ To $100 \mu \mathrm{g}$ (2 nmol as the dimeric lectin of Mol. Wt. of 50,000) of the purified basic pI-variant, dissolved in $100 \mu \mathrm{l}$ of 50 mM of HEPES ( $N$-[2-hydroxyethyl] piperazine- $N^{\prime}$ [2-ethanesulfonic acid]) - $\mathrm{NaOH}, \mathrm{pH} 7.5,1 \mu 1$ of 25 mM TR-succinimidyl ester in dimethylformamide was added and the reaction was allowed to proceed for $1.5 \mathrm{~h}$ at room temperature. The mixture was then passed through a small column of BioGel P6 equilibrated with $10 \mathrm{~mm}$ Tris-acetate, $\mathrm{pH} 7.9$, con-

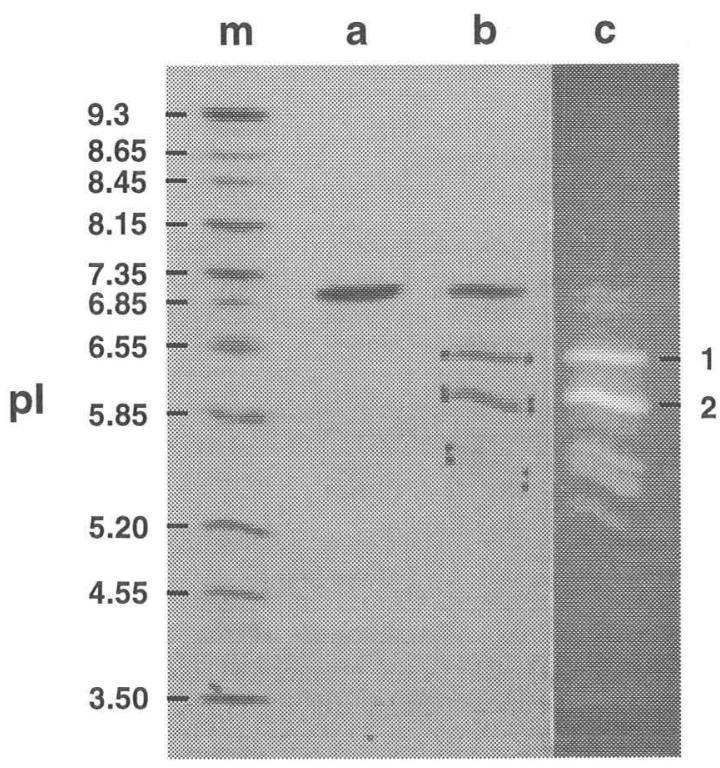

Fig. 1. Isoelectric focusing of pea lectin and the tetramethylrhodamine-labeled lectin.

Purified pea lectin and the product of its labeling with carboxytetramethylrhodamine succinimidyl ester were separated by isoelectric focusing on a polyacrylamide-gel slab. Lane a, purified pea lectin (protein staining); lane b, labeled product (protein staining; both sides of the bands are marked with dots based on visual inspection of fluorescence under illumination with a UV lamp prior to staining) ; lane c, a fluorescence photograph of lane b taken before staining; and lane $\mathrm{m}, \mathrm{pI}$ markers. taining $0.02 \% \mathrm{NaN}_{3}$. The fluorescent fraction (250 $\mu \mathrm{l})$ was collected under illumination by UV light and concentrated by means of a centrifugal evaporator to $20 \mu \mathrm{l}$. The concentrate was applied to a polyacrylamide-gel slab $(4.2 \% \mathrm{~T}, 4.8 \% \mathrm{C}, 55 \times 120 \times$ $0.5 \mathrm{~mm}$ thick, containing $2.5 \%$ carrier ampholyte, $\mathrm{pH} 3.5-10)$ with the electrodes placed on the shorter edges of the gel slab. Isoelectric focusing (IEF) was carried out at 100, 200, 400, 800 and, finally $1000 \mathrm{~V}$ each for $15 \mathrm{~min}$ to complete the migration of fluorescent bands. A gel piece containing the fluorescent basic band, corresponding to band 1 in lane $\mathrm{c}$ of Fig. 1, was excised with a knife and the labeled lectin was eluted twice by incubating the gel piece with $100 \mu \mathrm{l}$ of $0.1 \mathrm{M}$ Tris-acetate, $\mathrm{pH} 7.9$, containing $0.02 \% \mathrm{NaN}_{3}$. The concentration of the eluate, containing the pea lectin, which is labeled with a single dye (see below), was determined by the absorption measurement at $555 \mathrm{~nm}$ using a molar absorption coefficient of $64,000 \mathrm{~cm}^{-1} \mathrm{M}^{-1}$ for the dye. The yield of the labeled protein was $11 \mu \mathrm{g}$ based on 1-to-1 molar ratio of the dye to the dimeric lectin of $M_{r} 50,000$.

\section{Capillary affinophoresis}

The instrument for capillary electrophoresis, equipped with a laser-induced fluorescence detector has been described previously. ${ }^{3)}$ A capillary (50 $\mu \mathrm{m}$ i.d., $30 \mathrm{~cm}$ long, $10 \mathrm{~cm}$ from injection end to the detection point) coated with succinylpolylysine was placed in a jacket in which temperature-controlled water at $25^{\circ} \mathrm{C}$ was circulated. The column was filled with one of the affinophore solutions in the electrophoresis buffer ( $0.1 \mathrm{M}$ Tris-acetate, $\mathrm{pH} 7.9$, containing $0.02 \% \mathrm{NaN}_{3}$ ) by passing $20 \mu 1$ of the solution using a micro syringe connected to a Teflon tube. The solution of the labeled pea lectin (about $2.5 \mathrm{nl}$ containing $7.5 \mathrm{pg}$ ) was injected at the positive end by applying negative pressure $(-0.02 \mathrm{~atm})$ at the opposite end for about 2s. Electrophoresis was carried out at $150 \mathrm{~V} / \mathrm{cm}$ with a current of $11 \mu \mathrm{A}$ using the electrophoresis buffer as an electrode solution. After each run, the capillary was rinsed with $20 \mu \mathrm{l}$ of $0.1 \mathrm{M}$ sodium carbonate, $\mathrm{pH} 10$, containing $1 \mathrm{M} \mathrm{NaCl}$ and $10 \mathrm{~mm}$ EDTA, and then refilled with the electrophoresis buffer with or without the affinophore. The coupling product of TR succinimidyl ester with Tris (TR-Tris) was purified by reversed-phase HPLC using conditions 
described previously ${ }^{11)}$ and was used as a neutral marker for electrophoresis by adding it to the labeled-lectin solution at a concentration of $2.5 \times$ $10^{-8} \mathrm{M}$.

\section{RESULTS}

\section{Labeling of pea lectin}

A pI variant of pea lectin was purified by anion exchange chromatography to a preparation showing a single band on IEF in a polyacrylamide-gel slab at pH 7.05 (Fig. 1, lane a) and used for the labeling procedures. Tetramethylrhodamine contains one positive charge and one negative charge in the $\mathrm{pH}$ range from weakly acidic to alkaline, and thus, its net charge is zero over a relatively wide $\mathrm{pH}$ range, including the neutral $\mathrm{pH}$ region. Its carboxyl derivative, activated as a succinimidyl ester, was used for the labeling of the lectin.

The labeling reaction would be expected to occur largely at the $\varepsilon$-amino group of lysine residues and $\mathrm{N}$-terminal amino groups. This causes a reduction in positive charges, depending on the number of labels introduced, as a result of the formation of amide bonds. Therefore, at neutral $\mathrm{pH}$, a reduction of one positive charge would be expected for each label added, which results in the formation of a protein charge ladder. ${ }^{8)}$ Such differences in the number of labels are also represented as a pI ladder in the polyacrylamide-gel-slab IEF analysis (Fig. 1, lane c) and in capillary IEF analysis. ${ }^{11)}$ The most basic fluorescent band (Fig. 1, lane c, band 1), shifted to the acidic side by one step from the original protein, most likely represents the single-dyelabeled protein and the next fluorescent band (band 2 ), appearing as a doublet corresponds to the protein labeled with two dye molecules. These data are consistent with that obtained by capillary IEF, in which the singly labeled protein appeared as a single peak with a shoulder, and the doubly labeled protein appeared as a doublet with a shoulder. ${ }^{11)}$ The origin of such microheterogeneity is not yet clear, but is most probably due to site differences of labeling or the impurity of the reactive dye used for the labeling reaction. ${ }^{11}$ ) To exclude heterogeneity originating from a difference in the number of labels, the singly labeled pea lectin was purified by IEF in a polyacrylamide-gel slab and used in the following analysis by affinophoresis.

\section{Affinophoresis of fluorescence-labeled pea lectin}

The affinophoresis of labeled pea lectin was carried out in a covalently coated capillary with succinylpolylysine, a negatively charged polymer. The coating was found effective in suppressing the adsorption of a lectin, concanavalin A, and the consequent change in electroosmosis. ${ }^{3)}$ We experienced considerable changes of electroosmotic flow in first

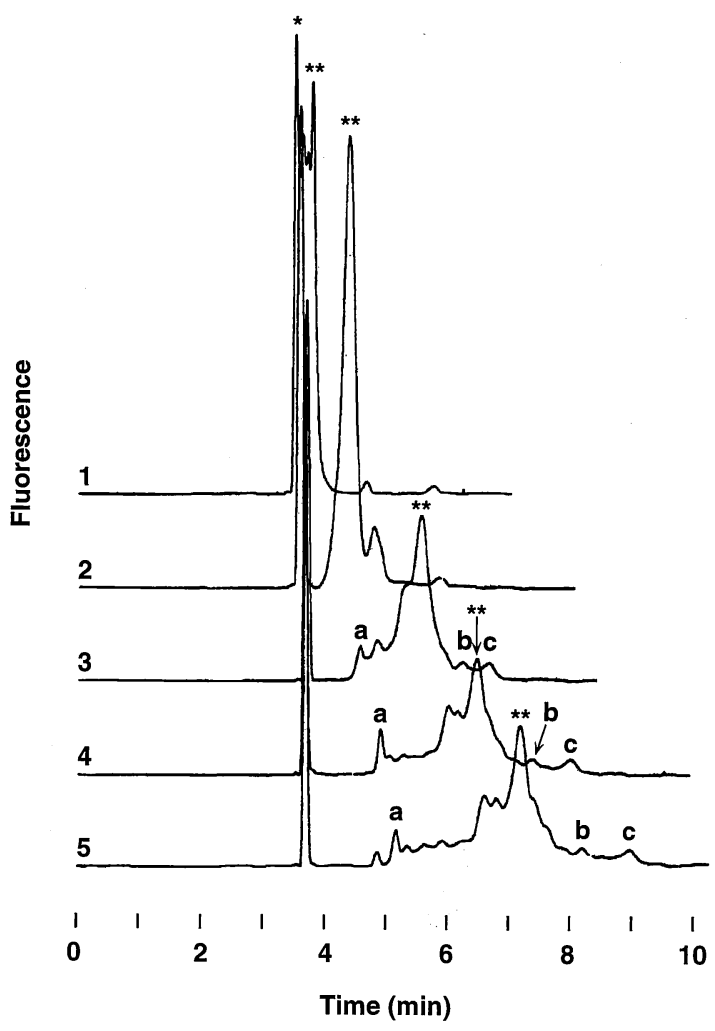

Fig. 2. Capillary affinophoresis of the fluorescencelabeled pea lectin in the presence of various concentrations of the mannoside-bearing affinophore, affinophore-1.

A fused silica capillary (50 $\mu \mathrm{m}$ i.d., $30 \mathrm{~cm}$ long), coated on the inside with succinylpolylysine, was filled with a solution of affinophore-1 dissolved in electrophoresis buffer $(0.1 \mathrm{~m}$ Tris-acetate, $\mathrm{pH} 7.9$,$) , and 7.5 \mathrm{pg}$ of the labeled pea lectin was injected at the positive end. Electrophoresis was carried out at $150 \mathrm{~V} / \mathrm{cm}(11 \mu \mathrm{A})$ and the labeled lectin was detected at a point $10 \mathrm{~cm}$ from the injection end with a LIF detector. The concentration of affinophore- 1 as the mannoside residue was: 1 , none ; $2,16.5 \mu_{\mathrm{M}} ; 3,49.5 \mu_{\mathrm{M}}$; $4,82.5 \mu \mathrm{M} ;$ and $5,116 \mu \mathrm{M}$. The main peak is marked $* *$ and the electrophoresis marker *. Minor peaks, analyzed for their affinity, are referred to as $a, b$ and $c$. 
Jpn J Electroph $1997 ; 41: 183$

two or three runs in a series of electrophoresis runs using uncoated fused silica capillaries but a capillary, coated with succinylpolylysine, consistently showed stable electroosmosis.

To the capillary, filled with the electrophoresis buffer containing the affinophore at different concentrations, the labeled lectin $(7.5 \mathrm{pg})$ was injected at the positive end with a fluorescent neutral marker, TR-Tris. Electrophoresis was carried out at a field strength of $150 \mathrm{~V} / \mathrm{cm}$ and the labeled lectin and TR-Tris were detected with LIF using $543.5 \mathrm{~nm}$ line of a He-Ne laser as the light source (Fig. 2). In the absence of the affinophore, the labeled lectin appeared as a bundle of peaks with small variations in electrophoretic mobility just behind the peak for TR-Tris $\left(^{*}\right)$. The addition of affinophore- 1 to the capillary caused an increase in the electrophoretic mobility of the labeled lectin towards the positive electrode, and thus, the peaks were detected at later times, as the concentration of the affinophore was increased. Since the addition of a haptenic ligand, methyl- $\alpha$-D-mannoside at $0.1 \mathrm{M}$, completely suppressed the effect of the affinophore, the mobility change observed in the presence of the affinophore can be attributed to the specific interaction between the labeled lectin and the affinity ligand of the affinophore. ${ }^{9)}$ Although the labeled pea lectin had been purified with respect of its $\mathrm{pI}$, the present result of zone electrophoresis demonstrated the presence of substantial heterogeneity in the labeled protein and the affinophoresis enhanced the separation of these components (Fig. 2).

\section{Quantitative analysis of the affinophoresis}

Since pea lectin is a divalent lectin and the affinophores are polyvalent, several possible forms of the lectin-affinophore complex would be expected from a 1-to-1 complex to a complex formed between multiple lectins and affinophores. Although we have no experimental information concerning the actual form of the complex under the present experimental conditions, as a first step for the analysis of such complex interactions, we assumed that all types of the complexes have the same electrophoretic mobility. The mobility change $(\Delta \mu)$ of the lectin caused by the affinophore (AP) can then be expressed by the following simple equation for affinity electrophoresis. ${ }^{4,12)}$

$$
\Delta \mu=\Delta \mu_{\max } \frac{[\mathrm{AP}]}{K_{\mathrm{d}}+[\mathrm{AP}]}
$$

where $\Delta \mu_{\max }$ is the maximum mobility change that corresponds to the mobility difference between free lectin and the complexes. The $K_{\mathrm{d}}$ is the overall dissociation constant for the lectin-affinophore complex, irrespective of the types of complexes, i.e., $K_{\mathrm{d}}$ $=[$ lectin $][\mathrm{AP}] /[$ complexes]. Equation $(1)$ can be rearranged to the equation below, which allows the determination of $K_{\mathrm{d}}$ and $\Delta \mu_{\max }$ by a linear plot of $\Delta \mu$ vs. $\Delta \mu /[\mathrm{AP}]$ from the slope and the intercept with the ordinate, respectively. ${ }^{5)}$

$$
\Delta \mu=\Delta \mu_{\max }-K_{\mathrm{d}} \frac{\Delta \mu}{[\mathrm{AP}]}
$$

The mobility change $(\Delta \mu)$ of the main peak and several minor peaks of the labeled lectin was analyzed for the interaction with affinophore-1 using Eq. 2 , where the concentration of the affinophores was expressed as the concentration of the mannoside residue (Fig. 3). The value of $\Delta \mu$ was calculated from the detection time for the peaks of the labeled lectin and the electrophoresis marker by using the equation described before. ${ }^{3)}$ The slowly migrating peak, peak a, had a slightly weaker affinity $\left(K_{\mathrm{d}}=\right.$ $80 \mu \mathrm{M})$ than the main peak $\left(* *, K_{\mathrm{d}}=67 \mu \mathrm{M}\right)$ to affinophore-1, whereas the maximum mobility change was approximately $50 \%$ of the main peak. The rapidly migrating two peaks, peak $\mathrm{b}$ and $\mathrm{c}$,

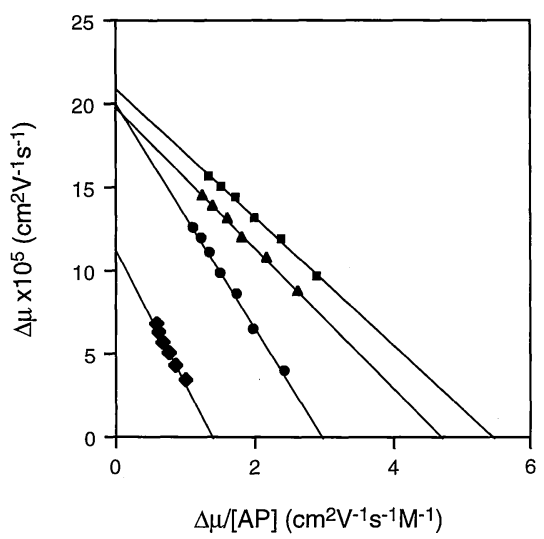

Fig. 3. Determination of the parameters of affinity electrophoresis for the main peak and minor peaks of the labeled pea lectin interacting with affinophore-1.

Linear plots were constructed, from the results partially presented on Fig. 2, according to Eq. 2 for the main peak $(\boldsymbol{O})$, peak a $(\boldsymbol{\bullet})$, peak b $(\boldsymbol{\Delta})$, and peak c $(\boldsymbol{\square})$. 


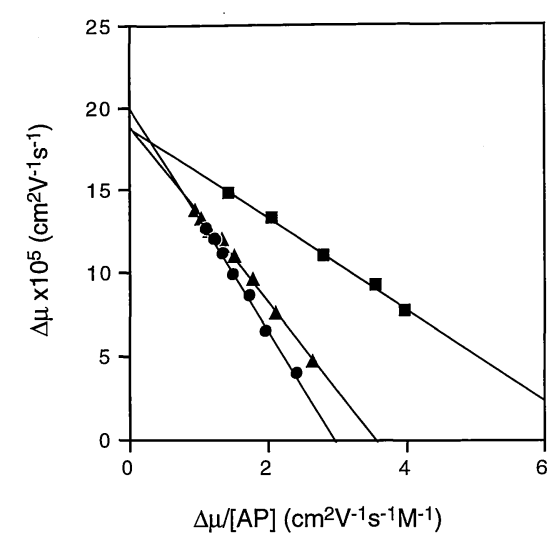

Fig. 4. Determination of parameters for affinity electrophoresis of the main peak of the labeled lectin interacting with the affinophores that are different in the density of the affinity ligand.

Linear plots were constructed according to Eq. 2 for affinophoresis with affinophore-1 (O), $-2(\mathbf{\Delta})$ and $-3(\mathbf{\square})$.

Table 1. Affinity-electrophoresis parameters for labeled pea lectin for the three affinophores.

\begin{tabular}{cccc}
\hline Affinophore & $\begin{array}{c}\text { Ligand density } \\
\text { (Man/Lys, \%) }\end{array}$ & $K_{\mathrm{d}}(\mathrm{mm})$ & $\begin{array}{c}\Delta \mu_{\max } \\
\left(\mathrm{cm}^{2} \mathrm{~V}^{-1} \mathrm{~s}^{-1}\right)\end{array}$ \\
\hline 1 & 4.2 & 0.067 & 20.1 \\
2 & 9.3 & 0.053 & 18.8 \\
3 & 17.5 & 0.027 & 18.7 \\
\hline
\end{tabular}

showed a slightly higher affinity for affinophore-1 than the main peak, i.e., $K_{\mathrm{d}}=42 \mu \mathrm{M}$ and $38 \mu \mathrm{M}$ for peak $\mathrm{b}$ and $\mathrm{c}$, respectively. The maximum mobility changes of the two components were nearly the same as that of the main peak.

The values of $K_{\mathrm{d}}$ and $\Delta \mu_{\max }$ were determined for the main peak with each of the affinophores which differed in the density of the affinity ligand (Fig. 4). These results are summarized in Table 1 . The affinity of the affinophore for the lectin increased as the density of the affinity ligand increased, whereas the difference in the maximum mobility change was quite small. These values are in good agreement with the values for the unlabeled pea lectin, determined by the same type of experiments using UVabsorption detection, i.e., $63 \mu \mathrm{M}$ for affinophore-1, 44 $\mu \mathrm{M}$ for affinophore- 2 and $26 \mu \mathrm{M}$ for affinophore-3 (unpublished results).

\section{DISCUSSION}

The affinophoresis of pea lectin using the polyligand affinophores was effective in the capillaries, as well as in agarose-gel slabs as has been reported previously. ${ }^{9}$ ) The labeling of the lectin with a fluorescent dye allowed a single run of the affinophoresis using $7.5 \mathrm{pg}$ of protein with a very large signal-to-noise ratio. We estimate that the enhancement in sensitivity over UV detection was several hundred fold as a result of the LIF detection. The labeling of protein with a fluorescent dye can thus significantly reduce the consumption of the protein, even in the case where the yield of labeled product is not very high.

A major problem in the use of fluorescence labeling was the unexpected heterogeneity in the mobility of the labeled product, even after purification by IEF. This makes a sharp contrast with the charge modification by acetylation. In the case of this small substituent, protein molecules having the same number of acetyl group but at different sites were not discriminated by affinity capillary electrophoresis. ${ }^{8)}$ The dissociation constants obtained for the three minor peaks of labeled lectin showed that two of these have slightly higher affinities, with the other a slightly lower one than the main peak. Despite such heterogeneity, the $K_{\mathrm{d}}$ values and the maximum mobility changes determined for the main peak showed good agreement with those determined for the native lectin by the same type of experiment using UV-absorption detection. We explain these results as follows. If it is assumed that the protein is spherical and that the labeling reaction occurs uniformly on the protein surface, at least a half of the labels will be introduced on the hemisphere removed from the binding site and only a small portion of the labeled protein molecules will contain the label at the area sufficiently close to the binding site to directly affect the binding characteristics. Therefore, the major components of the labeled products would be expected to retain their original binding characteristics.

Although fluorescence-labeling of a protein to achieve highly sensitive detection with LIF causes a considerable heterogeneity, the present results appears to justify the use of the labeled protein for 
the analysis of molecular interaction by means of affinity electrophoresis, where the efficient concomitant separation of the sample allows the discrimination of the major component of the labeling product from minor components, which might have an altered binding affinity. Farther investigation is necessary to see the general applicability of this approach.

\section{REFERENCES}

1) Chu Y-H, Avila LZ, Gao J, Whitesides GM. Affinity capillary electrophoresis. Acc Chem Res $1995 ; 28$ : 461-8.

2) Heegaard $\mathrm{NHH}$, Robey FA. The emerging role of capillary electrophoresis as a tool for the study of biomolecular noncovalent interactions. American Laboratory 1994 ; June issue : 28T28X.

3) Shimura K, Kasai K. Determination of the affinity constants of concanavalin A for monosaccharides by fluorescence affinity probe capillary electrophoresis. Anal Biochem 1995; 227 : 186-94.

4) Shimura K, Kasai K. Affinophoresis : Selective electrophoretic separation of proteins using specific carriers. In: Karger BL, Hancock, WS, editors. Methods Enzymol Vol. 271. High resolution separations and analysis of biological macromolecules part B, Chap 9. San Diego : Academic Press, 1996 : 203-218.
5) Shimura K, Kasai K. Determination of the affinity constants of pea lectin for neutral sugars by capillary affinophoresis with a monoligand affinophore. J Biochem 1996; 120 : 1146-52.

6) Yeung ES, Wang PL, Li W, Giese RW. Laser fluorescence detector for capillary electrophoresis. J Chromatogr 1992; 608 : 73-7.

7) Chen DY, Adelhelm K, Cheng XL, Dovichi NJ. A simple laser-induced fluorescence detector for sulforhodamine 101 in a capillary electrophoresis system : detection limits of 10 yoctomoles or six molecules. Analyst $1994 ; 119: 349-52$.

8) Gao J, Mammen M, Whitesides GM. Evaluating electrostatic contributions to binding with the use of protein charge ladders. Science 1996; $272: 535-7$.

9) Shimura K, Kasai K. Affinophoresis of pea lectin and fava bean lectin with an anionic affinophore, bearing $p$-aminophenyl $\alpha$-D-mannoside as an affinity ligand. J Chromatogr 1987; 400:353-9.

10) Trowbridge IS. Isolation and chemical characterization of a mitogenic lectin from Pisum sativum. J Biol Chem 1974; 249 : 6004-12.

11) Shimura K, Kasai K. Fluorescence-labeled peptides as isoelectric point (pI) markers in capillary isoelectric focusing with fluorescence detection. Electrophoresis $1995 ; 16: 1479-84$.

12) Takeo K. Advances in affinity electrophoresis. J Chromatogr A $1995 ; 698:$ 89-105. 